\title{
CIEN AÑOS DE LA SOCIEDAD ALEMANA DE ORQUIDEOLOGÍA (1906-2006)
}

\author{
Carlos O. Morales \\ Escuela de Biología, Universidad de Costa Rica, 2060 San José, Costa Rica, oldem@biologia.ucr.ac.cr
}

ABSTRACT. Hundred years of history of the German Orchid Society are here summarized.

Resumen. Se resumen cien años de historia de la Sociedad Alemana de Orquideología.

Palabras clave / Key words: Sociedad Alemana de Orquideología / Deutsche Orchideengesellschaft (D.O.G.)

Cuando una organización se funda para un fin específico, a menudo una serie de factores y eventos contribuyen para alimentar el caos y la entropía que, con el tiempo, ejercen una enorme resistencia que impide la persistencia de la organización. En gran parte debido a ello es que muy pocas entidades alcanzan varias décadas, más difícil aún, siglos de existencia, superando ingentes obstáculos.

Tal es el caso de la Sociedad Alemana de Orquideología (Deutsche Orchideengesellschaft o D.O.G.), que alcanzó este año su centésimo aniversario después de haber superado dos guerras mundiales, una crisis económica mundial, una guerra fría y múltiples avatares que el mundo ha sufrido a lo largo de este último siglo tan intenso, tan oscuro y, a la vez, tan luminoso.

En octubre de 1905 se reunieron varios orquidófilos en Berlín, con el objetivo de „unir a los orquidófilos alemanes en una sociedad y editar una revista, en la que ciencia y praxis de igual modo se expresen". Entre ellos estaban algunos nombres imborrables en la historia de la botánica, tales como el Prof. Dr. Udo Dammer, del Jardín Botánico de Berlín-Dahlem (que fue quien tuvo la iniciativa), y el Prof. Dr. Ernst Pfitzer, de Heidelberg, uno de los más grandes sistemáticos de orquídeas. En diciembre del mismo año se decidió fundar la sociedad y la primera junta directiva se nombró el 10 de mayo de 1906 en Berlín, presidida por el Barón Max von Fürstenberg, quien llegó a tener una colección de más de 2000 especies de orquídeas, de la cual se sirvió Schlechter para su libro "Die Orchideen"; miembro de esta junta fue también Bruno Fritsch (Fig. 1), un eminente hombre de la política y apasionado de la naturaleza, que tuvo un puesto administrativo en el Parque Zoológico de Berlín, fue miembro honorario de la Sociedad Alemana de Horticultura y todavía en
1925, con 85 años de edad, fue nombrado de nuevo en la junta directiva de la Sección de Orquideología. Los otros miembros de la primera junta fueron Otto Beyrodt, Udo Dammer y Paul Herz; en un comité de consejeros se encontraba Ernst Pfitzer. Al mismo tiempo tuvo lugar una exposición, visitada por unas 700 personas, en la que participaron famosos jardineros de la época, pero también orquidófilos privados.

El primero de abril de 1906 apareció el primer número de Orchis, revista mensual de la Sociedad Alemana de Orquideología, editado por Dammer. Un vasto interés en las orquídeas del mundo se refleja ya en el primer número, en el que aparece un artículo sobre las orquídeas del Kilimanjaro, otro en lengua francesa sobre la importancia de los hongos simbióticos de las orquídeas, varios artículos referentes al cultivo de orquídeas y la reseña de un libro. Desde el primer número se publican láminas en colores, lo que hace a la revista muy atractiva hasta la actualidad. El segundo número salió a la luz el primero de mayo de 1906. En el tercer número se publica una lista de 241 miembros, entre ellos el jardinero e importador de orquídeas Robert Blossfeld, el jardinero y cazador de orquídeas Hermann Sandhack, el botánico Rudolf Schlechter, el coleccionista Walter Rothschild, de Londres, y los jardineros ingleses Sander \& Sons. El primer número del segundo volumen de Orchis se publicó en octubre de 1907; el nuevo editor era Friedrich Kränzlin (Fig. 1), uno de los principales expertos en orquídeas de inicios del siglo XX, quien simplificó el diseño, redujo el tamaño, no incluyó láminas en colores, pero incluyó más contribuciones científicas.

En 1908 la Sociedad Alemana de Orquideología se convirtió en una sección de la Sociedad Alemana de Horticultura (Deutsche Gartenbau-Gesellschaft o D.G.G.). La Sección de Orquideología (Orchideen- 
Abteilung) nombró un comité, que se reunió regularmente cada mes entre 1908 y 1920, siempre organizando exposiciones y juzgamientos de plantas. En 1909, el Prof. Kränzlin se ofreció para determinar orquídeas críticas o desconocidas, lo que en la actualidad realiza un Centro de Identificación de Orquídeas. En este año, la revista de orquideología apareció como suplemento de „Gartenflora“, el órgano de la D.G.G. Muy significativo en la historia de la botánica es que en 1911 el Dr. Rudolf Schlechter (Fig. 1), uno de los cuatro más grandes orquideólogos de todos los tiempos, se convirtió en secretario del Comité de Orquideologia y en 1914 en editor de la revista Orchis. En Lankesteriana 6: 29-30, 2003, se publicó un resumen de la biografía de Schlechter, a partir de la que plasmó K. Senghas en Schlechter: Die Orchideen, 3. Auflage, 2002.

Desde 1906 hasta el inicio de la Primera Guerra Mundial (1914) se realizaron exposiciones regularmente, la mayoría en Berlín. Alrededor de 1910, las exposiciones ya contaban con iluminación eléctrica, sobre todo en las noches. A partir de 1913, en las exposiciones se presentaron libros y en las paredes se colgaron fotos de grandes dimensiones. A finales de 1914 empezó la Primera Guerra Mundial, por lo que se acabaron las exposiciones; sin embargo, el Comité de Orquideología siguió reuniéndose. En septiembre de 1915 Schlechter fue llamado a prestar servicio militar; en 1917 regresó a casa debido a una herida. Aunque la guerra terminó en 1918, en los difíciles años de posguerra muchas colecciones y viveros de orquídeas debieron ser clausurados por dificultades financieras, falta de personal, de materiales para calefacción, etc. En ese año hubo ocho en lugar de 12 reuniones del comité; en 1919 solamente cinco reuniones, lo que refleja una situación muy difícil.

Entre 1920 y 1924 los orquidófilos siguieron reuniéndose, pero en períodos irregulares. Las razones principales fueron: una difícil situación económica (porque Alemania tuvo que pagar grandes sumas por reparaciones de guerra a las potencias vencedoras), medios de transporte entre Berlín y alrededores suspendidos y el presidente del Comité de Orquideología, Otto Beyrodt, estaba enfermo y murió en 1923. La revista Orchis apareció por última vez en abril de 1920; no obstante, mediante breves boletines y después por medio de una crónica sumaria de los eventos de la sociedad, los miembros pudieron informarse apropiadamente sobre todos los sucesos y los esfuerzos realizados. Entre 1922 y 1924 no se publicó ninguna revista, ni siquiera Gartenflora, debido a la grave situación económica con una inflación insoportable. Lammers $(2003)^{1}$ menciona una cifra espeluznante: el 20 de noviembre de 1923 el valor del marco alemán tocó fondo y un dólar equivalía a 4,2 billones de marcos (4 200000000000$)$.

Desde febrero de 1924 se publicó de nuevo Gartenflora, de modo regular, con avisos sobre reuniones de la Sección de Orquideología, así como informes y artículos sobre orquídeas. En 1925 se nombró un nuevo Comité de Orquideología, que a partir de este año se reunió mensualmente en el Museo Botánico de Berlín-Dahlem. Significativamente, un nuevo miembro del comité fue el Prof. Rudolf Mansfeld, quien trabajó en el Jardín Botánico y en el Museo Botánico de Berlín entre 1926 y 1943; además, fue el editor de "Schlechter: Figurenatlas zu den Orchideenfloren der südamerikanischen Kordillerenstaaten" y colaborador en la 3a. edición de "Schlechter: Die Orchideen". En las reuniones mensuales de 1925 se iniciaron conferencias con diapositivas, se presentaron plantas para ser juzgadas y se divulgaron nuevos libros sobre orquídeas. Hasta 1933, la Sección de Orquideología trabajó activamente, después sólo de modo esporádico. Esto último es indicador de que, de nuevo, había una gran turbulencia política, economía y social en Alemania.

En 1937 se fundaron los primeros grupos regionales; por ej. Berlín, Bremen, Hamburgo, Múnich y Dresde. De nuevo se realizaron reuniones mensuales y reuniones anuales y la Sección de Orquideología volvió a ser muy activa; se ofrecieron conferencias con diapositivas en colores y se creó un centro de investigación sobre orquídeas, que contemplaba la protección de especies y la reproducción de las que estaban en peligro de extinción, entre otros aspectos. En este año se publicó de nuevo la revista Orchis; en 1938 apareció con papel e ilustraciones de gran calidad, incluso en colores. Entre 1938 y 1941 la revista salió a la luz mensualmente de modo regular, pero desde 1939 se publicó cada tres meses. El nuevo desarrollo alcanzado con gran esfuerzo por los orquidófilos alemanes después de la Primera Guerra Mundial encontró de nuevo un final dramático, pues tras el anuncio „Seit heute wird jetzt

${ }^{1}$ Lammers, Rebecca. 2003. 15. November 1923. Die Inflation wird mit der Einführung der Rentenmark gestoppt. In: Die Weimarer Republik, 1918-1933. http://www.nrw2000.de/weimar/rentenmark.htm 
zurückgeschossen!“ (¡Desde hoy se contestará el fuego!) el 1 1. de septiembre de 1939 empezó la Segunda Guerra Mundial.

A partir de julio de 1942 no se permitió la publicación de ninguna revista o escrito similar. Por eso se editaron circulares llamadas Orchideenbriefe (cartas de orquídeas), que aparecieron todavía en diciembre de 1944 en forma de revista. Desde 1942 no hubo más reuniones públicas de orquidófilos. Sin embargo, los miembros de los diversos grupos siguieron reuniéndose mensualmente, en la medida en que las limitaciones de la guerra lo permitían (malas condiciones de transportes, obligación de permanecer a oscuras, etc.; después destrucción por acciones bélicas de distritos enteros de ciudades). Es sorprendente la valentía y la persistencia de los orquidófilos de Hamburgo, que lograron reunirse regularmente todavía hasta mediados de 1944, cuando la destrucción causada por la guerra llegaba a su extremo.

La guerra terminó el 8 de mayo de 1945, pero vinieron tiempos muy difíciles; muchas personas padecieron problemas de hambre, casas y edificios destruidos, toque de queda, reforma monetaria, etc. Igual que muchas casas, también muchos invernaderos fueron destruidos y la mayor parte de las orquídeas cultivadas en Alemania no sobrevivieron a la guerra. Aún así, ya en 1946 se reunieron de nuevo los orquidófilos hamburgueses y en septiembre de ese año decidieron fundar nuevamente una sociedad orquideológica, con permiso otorgado por el gobierno militar de entonces.

Entre noviembre de 1946 y finales de 1947 se enviaron boletines escritos a máquina para sustituir la revista. La primera reunión anual de los orquidófilos alemanes tuvo lugar en Hamburgo en marzo de 1948. La D.O.G. tenía ya 192 miembros alemanes y 34 extranjeros; la junta directiva estaba integrada por Julius Heinrich Müller, Carl Pretzel y Georg Vorwerk (Fig. 1). Fue hasta abril de 1949 cuando el Senado de la Ciudad Hanseática de Hamburgo otorgó permiso para fundar una revista. El primer número de "Die Orchidee" se publicó en octubre-diciembre de 1949. Desde ese año hubo de nuevo reuniones anuales y se fundaron numerosos grupos regionales, como los de Hamburgo, Berlín y Francfort del Meno. En esta última ciudad se realizó la reunión anual de 1950, por primera vez fuera de Hamburgo.

Los progresos fueron rápidos. Desde 1950 hubo de nuevo exposiciones de orquídeas y ya en 1960 la
D.O.G. estuvo representada de nuevo en un congreso mundial (en Londres). Desde 1962, el Prof. Dr. Wolfgang Haber ocupó la presidencia de la D.O.G. y desde entonces aumentaron las actividades científicas de modo evidente. En la reunión anual de 1962 se comunicó que la D.O.G. tenía 1350 miembros, 300 de ellos extranjeros. Desde 1963 hay de nuevo una exposición de orquídeas asociada a cada reunión anual. En 1973 se fundó una comisión de juzgamientos, que se mantiene activa mensualmente en diferentes lugares de Alemania y del extranjero; esta comisión realiza juzgamientos de plantas y puestos, incluso en exposiciones. Un evento trascendental en la historia de la D.O.G. fue la Octava Conferencia Mundial de Orquideología en abril de 1975, en el Palmengarten de Francfort del Meno.

También en la zona oriental de Alemania, ocupada por los soviéticos, hubo actividad orquidófila desde los tiempos de la posguerra. Es interesante saber que al principio los orquidófilos germanos orientales podían ser miembros de la D.O.G., participar en las reuniones de ésta y recibir la revista; sin embargo, desde 1961, cuando se construyó el muro de Berlín, los alemanes orientales no tuvieron autorización para visitar exposiciones ni congresos en Alemania occidental; si ellos deseaban publicar en la revista Die Orchidee, debían solicitar un permiso, que a menudo no era otorgado. A pesar de todo, en 1963 se fundó en Greifswald, Alemania oriental, un grupo de trabajo de orquideología. Desde ese año, se formaron grupos regionales, hubo exposiciones y reuniones y desde 1968 se publicó la revista Orchideen. En la década de 1980 había 42 grupos regionales con unos 1300 miembros.

En 1990, después de la reunificación de los dos estados alemanes, se fusionaron también ambas sociedades de orquideología y muchos miembros de grupos alemanes orientales se convirtieron en miembros de la D.O.G. En 1991 se nombró un nuevo equipo de redacción para la revista; asimismo, se inició el trabajo de un centro de identificación de orquídeas. En 1993 Gerd Röllke fue nombrado presidente de la D.O.G. y desde entonces hasta hoy ha sido reelegido repetidas veces. En 1996 la D.O.G. adquirió una sede fija en Stukenbrock, cerca de Bielefeld, que incluye la biblioteca. Desde entonces, los consejos de la junta directiva y las reuniones editoriales tienen lugar allí.

La D.O.G. realiza numerosas actividades durante casi todo el año en diversos lugares de Alemania y 
participa frecuentemente en congresos y exposiciones internacionales. En abril de 2006, con motivo del simposio anual de orquídeas en Dresde, aunado a una gran exposición internacional de orquídeas, se celebraron los 100 años de la D.O.G. con muchos invitados del país y del extranjero.

En la actualidad, la Sociedad Alemana de Orquideología cuenta con más de 6000 miembros, entre ellos muchos en el extranjero, sobre todo en EE.UU. (Bärbel Röth, com. pers., oct. 2006). La D.O.G. muestra una larga y persistente trayectoria, que es un ejemplo a nivel mundial. Con toda seguridad, la experiencia adquirida por esta sociedad podría ser muy provechosa para otras sociedades más recientes, sobre todo en países más jóvenes del mundo, que actualmente intentan organizar grupos y actividades para proteger el ambiente e interesar a los ciudadanos comunes en la naturaleza. Existen notables evidencias de que las orquídeas son un indicador muy seguro de la calidad del ambiente y, por tanto, pueden ofrecer mucha información acerca de lo que está sucediendo en diversos ecosistemas naturales.

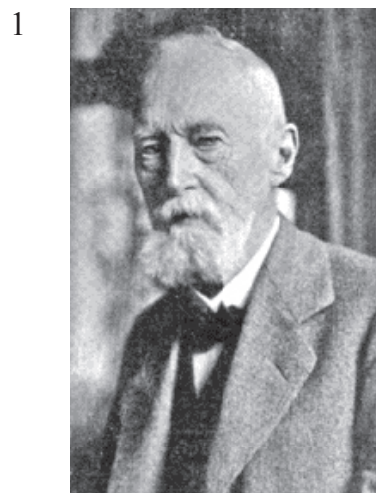

3

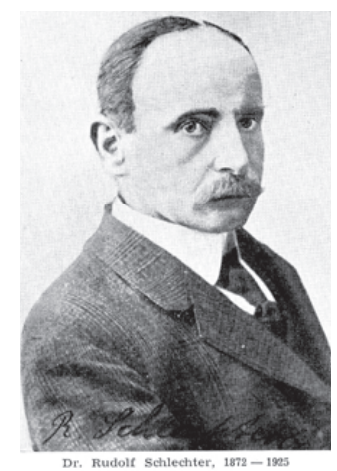

4
Asimismo, las orquídeas pueden jugar un papel importante en los procesos de educación ambiental, que intentan despertar la conciencia de cada vez más ciudadanos del mundo, para revertir las actividades antropógenas destructivas, que tanto daño han causado en todo el planeta.

Para los lectores que conocen la lengua alemana, es recomendable Die Orchidee 47(2) de marzo-abril de 1996, un número especial con motivo del nonagésimo aniversario de la D.O.G., en el que Gerd Röllke, Bärbel Röth y Jürgen Röth presentan detalladamente la historia de la sociedad. Algunos comentarios sobre los 100 años de la D.O.G. también se hallan en Die Orchidee 57(1) de 2006.

Agradecimiento. A Bärbel Röth (D.O.G.), quien en septiembre y octubre de 2006 me envió información valiosísima y fotos del archivo de la Sociedad Alemana de Orquideología. Sin ese material único no habría sido posible concluir este escrito.

2
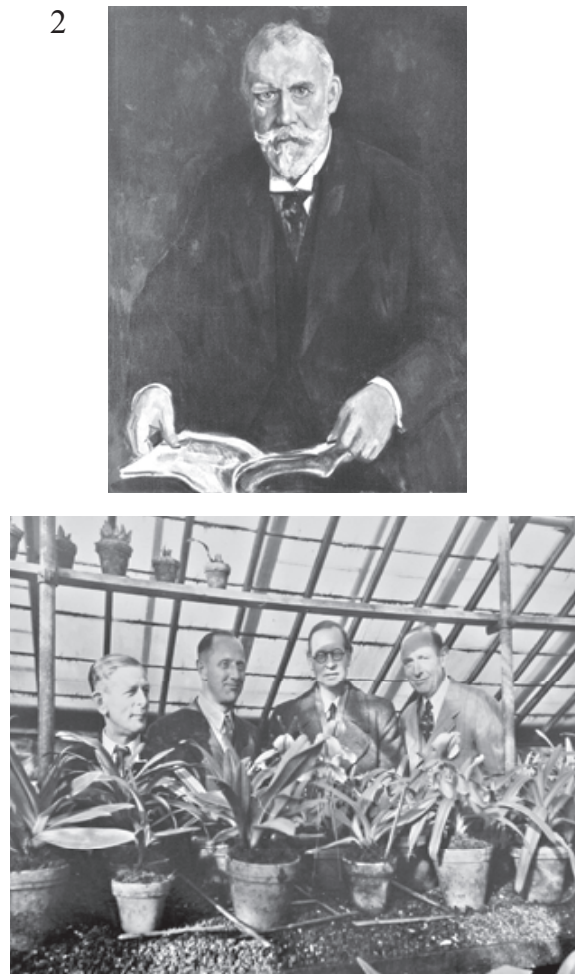

Fig. 1. Algunas de las personas que jugaron un papel extraordinario en la historia de la Sociedad Alemana de Orquideología (D.O.G.). 1. Bruno Fritsch, 1906; 2. Friedrich Kränzlin, ca. 1920; 3. Rudolf Schlechter, 1924; 4. Después de la devastación de la Segunda Guerra Mundial, los nuevos fundadores de la D.O.G. en 1946; de izquierda a derecha: el asesor Albert Springer y los miembros de la junta directiva Julius Heinrich Müller, Georg Vorwerk y Carl Pretzel. Fotos del archivo de la D.O.G., cortesía de Bärbel Röth. 